\title{
UTILIZAÇÃO DE CINZAS DA INCINERAÇÃO DE APARAS DE COURO NA PRODUÇÃO EM LABORATÓRIO DE FeCr-AC
}

\author{
Claudia Rosane Ribeiro Alves ' \\ Nestor Cezar Heck ${ }^{2}$ \\ Ivo André Homrich Schneider ${ }^{3}$
}

\section{Resumo}

A indústria do couro produz aparas durante o curtimento do couro que, por causa do método empregado, têm cromo na sua composição química. $O$ volume das aparas pode ser reduzido e o seu conteúdo energético pode ser aproveitado com a incineração. O objetivo deste trabalho é mostrar que é possível a reutilização do metal contido nas cinzas das aparas incineradas como matéria-prima na produção de aço inoxidável, livrando o meio ambiente de um resíduo perigoso. Para isso, foi investigada em laboratório a elaboração de uma liga com as características similares às de uma ferroliga FeCr-AC comercial. Uma análise termodinâmica simulando o processo de redução do $\mathrm{Cr}_{2} \mathrm{O}_{3}$, com o objetivo de fundamentar e de fornecer as melhores condições para os experimentos, foi realizada em outro momento. Ao nível de laboratório, ficou demonstrado que é possível a reutilização do cromo contido nas cinzas da incineração das aparas de couro para a finalidade prevista.

Palavras-chave: Cromo; Ferro-ligas; Resíduos; Couro.

\section{UTILIZATION OF ASHES FROM THE LEATHER SHAVINGS INCINERATION FOR THE PRODUCTION OF HC-FeCr ALLOY IN LABORATORY SCALE}

\begin{abstract}
The tanning industry, during the leather tanning, produces shavings that, because of the method used, have chromium in its chemical composition. Volume reduction and energy utilization can be achieved by shavings incineration. The aim of this work is to show that the recycling of the metal contained in leather shaving ashes is possible as raw material for stainless steel production, relieving the environment of a dangerous residue. The elaboration of a ferroalloy with similar characteristics of the commercial $\mathrm{HC}-\mathrm{FeCr}$ ferroalloy was investigated at the laboratory scale. Prior, a thermodynamic analysis was carried out, simulating the process of chromium oxide reduction in order to provide the basis and the most favorable conditions for the experiments. At the laboratory level, it was demonstrated that is viable the reutilization of chromium bearing ashes from the incineration of leather shavings for the production of $\mathrm{HC}-\mathrm{FeCr}$.
\end{abstract}

Key words: Chromium; Ferroalloy; Residues; Tanning.

\section{INTRODUÇÃO}

Foram produzidas no ano de 2004 , no Brasil, somente no setor calçadista, 166 mil toneladas de resíduos sólidos. Deste total, $40 \%-50 \%$ correspondem a resíduos contendo cromo em sua composição.

Frente à intensa conscientização ecológica - ao lado da legislação ambiental, cada vez mais rigorosa, que atinge tanto empresas que produzem resíduos quanto as que os recebem - é cada vez maior o interesse do setor produtivo em proteger o meio ambiente. Dessa conscientização resulta a necessidade de se desenvolver um processo de tratamento junto a esse ramo industrial, com o objetivo de reduzir o volume e, ao mesmo tempo, aproveitar o conteúdo energético do resíduo sólido contendo cromo - como alternativa para não aumentar o passivo ambiental que se estabelece quando tal resíduo é enviado aos aterros industriais de resíduos perigosos (ARIPs). Tal processo de tratamento consiste basicamente de uma unidade incineradora dotada de um sistema de tratamento de gases, com capacidade nominal de processar $100 \mathrm{~kg} / \mathrm{h}$ de resíduos

\footnotetext{
'Mestre, Aluna de Doutorado, Programa de Pós-Graduação em Eng. de Minas, Metalúrgica e de Materiais, PPGEM, LEAmet / NTCm, Centro de Tecnologia, UFRGS, Campus do Vale. Av. Bento Gonçalves, 9500, Cep9150I-970, Porto Alegre, RS, Brasil. E-mail: claudia.alves@ufrgs.br. ${ }^{2}$ Dr., Professor, Núcleo de Termodinâmica Computacional para a Metalurgia - NTCm. Departamento de Metalurgia, Centro de Tecnologia, UFRGS, Campus do Vale. Av. Bento Gonçalves, 9500, Cep 9150I-970, Porto Alegre, RS, Brasil. E-mail: heck@ufrgs.br. ${ }^{3}$ Dr., Professor, Laboratório de Estudos Ambientais para Metalurgia - LEAmet; Departamento de Metalurgia, Centro de Tecnologia, UFRGS, Campus do Vale. Av. Bento Gonçalves, 9500, Cep 9150I-970, Porto Alegre, RS, Brasil. E-mail: ivo.andre@ufrgs.br.
} 
sólidos. Esse equipamento, contudo, ao atingir estes objetivos, produz um resíduo sólido secundário - as cinzas da incineração de aparas de couro (CIAC), geradas numa proporção de $\sim 6,5 \mathrm{~kg}$ de CIAC por $100 \mathrm{~kg}$ de resíduo - ao qual se deve dar uma destinação (deve ser salientado que já foram processadas cerca de 200 toneladas do resíduo nesse equipamento, em 2.500 h de operação da usina $^{(l)}$ ).

Como as cinzas são ricas em óxidos de cromo (40\%-50\%, em massa), elas devem ser tratadas. Dentre as alternativas para $\circ$ aproveitamento desse metal aparecem tanto a indústria química especialmente os curtumes - quanto a metalúrgica.

No ramo metalúrgico, tem destaque a siderurgia como grande consumidora de cromo, utilizado na produção de aços inoxidáveis. Nesse caso, o metal pode ser introduzido no processo sob a forma de ferroliga ( $\mathrm{Fe}-\mathrm{Cr}$ ) com teor elevado de cromo. Com base em um balanço de massa, a massa de cromo contida em $100 \mathrm{~kg}$ de CIAC seria suficiente para a produção de cerca de $65 \mathrm{~kg}$ da ferroliga citada. ${ }^{(2)}$

A produção de $\mathrm{FeCr}-\mathrm{AC}$ (variedade de alto carbono) dá-se em fornos elétricos de redução (FER) revestidos com refratários magnesianos ou com uma pasta carbonosa. Segundo Beneduce Neto, ${ }^{(3)} \circ$ minério primário utilizado é tipicamente cromita $\left(\mathrm{FeO} \cdot \mathrm{Cr}_{2} \mathrm{O}_{3}\right)$, associada à qual ocorre uma ganga aluminomagnesiana, e a redução é levada a cabo pelo carbono. A escória, no processo industrial, tem a composição ajustada para que a temperatura liquidus se situe na faixa de $1.700^{\circ} \mathrm{C}$ a $1.800^{\circ} \mathrm{C}$.

O objetivo do presente trabalho é demonstrar a possibilidade de reutilização do cromo contido na CIAC como matéria prima para a obtenção de ferroliga $\mathrm{Fe}-\mathrm{Cr}-\mathrm{AC}$. Nos experimentos, a redução do $\mathrm{Cr}_{2} \mathrm{O}_{3}$ é efetuada por meio da redução carbotérmica da mistura de CIAC com carvão mineral contida em cadinhos, em fornos aquecidos por resistências elétricas.

As simulações termoquímicas que constituem os fundamentos do presente trabalho foram publicadas em outro artigo. ${ }^{(4)}$

\section{METODOLOGIA}

Os experimentos para a obtenção da ferroliga $\mathrm{Fe}-\mathrm{Cr}$ por redução carbotérmica da CIAC foram efetuados pela sua mistura com carvão e ferro em pó, dentro de cadinhos com capacidades distintas, em fornos aquecidos por resistências elétricas. A origem e as características das matérias primas, bem como os detalhes dos experimentos, são descritos a seguir.

\section{I Cinzas de Incineração de Aparas de Couro, CIAC}

As amostras da CIAC são provenientes da planta-piloto instalada junto à empresa Preservar Tratamento e Reciclagem de Resíduos Ltda., localizada no município de Dois Irmãos, RS. O resíduo sólido industrial é basicamente processado num reator de leito fixo com tecnologia de gaseificação e combustão combinadas (GCC). O reator de gaseificação opera à temperatura de $650^{\circ} \mathrm{C}$; nesta temperatura ocorrem: i) o processo de secagem do resíduo, ii) a perda de matéria volátil e iii) a produção de gases combustíveis.
A maior parte das cinzas gerada como subproduto do processo de tratamento provém do reator de gaseificação e fica depositada no cinzeiro localizado na sua base. Frações menores são coletadas nos ciclones e lavadores.

Por ter origem numa mistura de resíduos provenientes de diversas indústrias, e por ter diferentes naturezas, é de se esperar, contudo, que a CIAC apresente certa variação na sua composição.

\subsection{Carvão e Ferro}

O carvão foi fornecido pela Indústria Carbonífera Rio Deserto, localizada no estado de Santa Catarina. Ele foi submetido a um processo de cominuição, resultando num material com uma granulometria menor que $0,0250 \mathrm{~mm}$, com $86 \%$ de carbono fixo e $0,86 \%$ de enxofre total (em base seca).

O ferro utilizado era de pureza analítica, com um teor maior que $98 \%$.

\subsection{Produção da Ferroliga}

$\mathrm{Na}$ composição da carga manteve-se a proporção entre $\mathrm{CIAC}$, ferro e redutor determinada na simulação termodinâmica prévia da redução (ver adiante). A massa total das cargas, contudo, variou, para que estas se adaptassem às capacidades dos diferentes tipos de cadinhos de grafita empregados: tipo I: $3,4 \mathrm{~cm}$ de altura e $2 \mathrm{~cm}$ de diâmetro interno; tipo $2: 8 \mathrm{~cm}$ de altura e $3 \mathrm{~cm}$ de diâmetro interno; tipo $3: 9 \mathrm{~cm}$ de altura e $4 \mathrm{~cm}$ de diâmetro interno.

Para a produção da liga foram utilizados dois fornos elétricos resistivos de laboratório: um forno especialmente projetado para os experimentos (tipo tubo vertical) e outro tipo mufla. Enquanto que $o$ primeiro continha apenas um cadinho (tipos I ou 2), por vez o segundo podia conter vários deles (tipo 3), simultaneamente.

Os experimentos com cadinhos dos tipos I e 2 foram realizados à temperatura final de $1.600^{\circ} \mathrm{C}$. Argônio era injetado pela parte superior do forno a uma vazão de $5 \mathrm{~L} / \mathrm{min}$. As taxas de aquecimento do forno e o tempo de permanência na temperatura máxima foram as seguintes: $10^{\circ} \mathrm{C} / \mathrm{min}$ (de $20^{\circ} \mathrm{C}$ até $1.400^{\circ} \mathrm{C}$ ); $5^{\circ} \mathrm{C} / \mathrm{min}\left(\right.$ de $1.400^{\circ} \mathrm{C}$ até $\left.1.600^{\circ} \mathrm{C}\right)$ e 15 min à temperatura de $1.600^{\circ} \mathrm{C}$, para experimentos com cadinhos do tipo I; idem para experimentos com cadinhos do tipo 2 , exceto para 60 min no patamar a $1600^{\circ} \mathrm{C}$. As taxas de resfriamento para ambos foram: $5^{\circ} \mathrm{C} / \mathrm{min}$ (de $1.600^{\circ} \mathrm{C}$ até $1.400^{\circ} \mathrm{C}$ ) e $3^{\circ} \mathrm{C} / \min \left(\right.$ de $1.400^{\circ} \mathrm{C}$ até $1.100^{\circ} \mathrm{C}$ ). A CIAC e o carvão sofreram uma compactação prévia moderada; além disso, para alojar o ferro, 
foi feita uma cavidade central no cadinho, conforme apresentado na Figura I. Também foi utilizada uma camada de carvão como cobertura extra, para proteger a carga da ação do oxigênio da atmosfera. Nesses experimentos, as massas das cargas foram: 7,4 g e $87 \mathrm{~g}$, para cadinhos dos tipos I e 2, respectivamente.

Devido à restrição de volume imposta pelo forno, foram tentados alguns outros experimentos para a obtenção de maiores quantidades de liga (material fundido). Contudo, apenas aqueles correspondentes ao cadinho tipo 3 foram bem sucedidos, em um terceiro forno resistivo. A carga desse experimento variou entre $96 \mathrm{~g} \mathrm{e} 173 \mathrm{~g}$. A amostra foi mantida dentro da câmara por um período de $2 \mathrm{~h}$, até ser atingida a temperatura limite, de $1.300^{\circ} \mathrm{C}$. Esta temperatura foi mantida por $30 \mathrm{~min}$, após os quais o material foi resfriado, dentro do forno, até a temperatura ambiente.

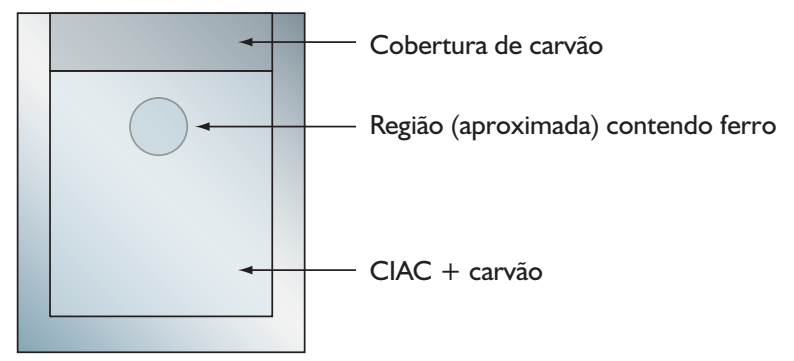

Figura I. Diagrama esquemático da distribuição aproximada da carga nos cadinho de grafita dos tipos I ( $3,4 \mathrm{~cm}$ de altura e $2 \mathrm{~cm}$ de diâmetro interno) e 2 ( $8 \mathrm{~cm}$ de altura e $3 \mathrm{~cm}$ de diâmetro interno).

\section{RESULTADOS E DISCUSSÃO}

\section{I Análise Termodinâmica}

A produção de um metal ou liga metálica, a partir de seus óxidos, com a utilização da pirometalurgia, normalmente envolve o emprego de processos de redução carbotérmica. Rosenqvist, ${ }^{(5)}$ por exemplo, apresenta de maneira didática os seus fundamentos.

A base da redução carbotérmica do óxido de cromo pode ser estudada com o auxílio do sistema simples Fe-Cr-O-C. Um estudo da redução, para esse sistema, à temperatura de $1.600^{\circ} \mathrm{Ce}$ pressão de I atm, foi realizado previamente ${ }^{(4)}$ com o auxílio de uma ferramenta termodinâmica computacional denominada FactSage. ${ }^{(6)}$ Apenas os resultados principais são repetidos aqui.

Com base nos resultados desse estudo, foi elaborada uma simulação que determinou que um composto com $100 \mathrm{~g}$ de CIAC, $3 \mathrm{l} \mathrm{g}$ de carbono e $45 \mathrm{~g}$ de ferro tem a composição ideal para os testes de laboratório. Para essa massa de reagentes, à temperatura de $1.600^{\circ} \mathrm{C}$ e pressão de $\mathrm{I}$ atm, não é mais esperada a existência de produtos sólidos. É importante que os produtos estejam no estado líquido no momento da produção da liga: isso favorece tanto o coalescimento das frações de metal produzido quanto o seu escoamento.

Resta salientar que, em comparação com o minério primário (cromita), a cinza não possui ganga associada, e é menos refratária. Isso provavelmente contribuirá para que a sua utilização, através desse processo, se torne viável na prática.

\subsection{Análise Experimental}

\subsection{Caracterização das Cinzas da Incineração de Aparas de Couro (CIAC)}

A Tabela I apresenta o resultado da análise elementar em cinzas de incineração de aparas de couro. Pode-se observar que, dentre os elementos analisados, o majoritário é o cromo. Já a concentração de $\mathrm{Cr}(\mathrm{VI})$, na cinza coletada no cinzeiro, conforme Godinho et al.,"(1) é inferior a 400 ppm em todas as amostras analisadas. A presença de carbono e nitrogênio é bastante baixa, indicando que o processo de combustão foi muito eficiente. Gómez-Moreno, ${ }^{(7)}$ citado em Godinho et al.,(1) salienta que a concentração de carbono inferior a $0,7 \%$ em cinzas sugere a boa combustão do material a partir do qual elas se originaram.

As fases cristalinas presentes em amostra de CIAC foram determinadas por difração de raios $X$, Tabela 2. Foi identificada a presença de eskolaita $\left(\mathrm{Cr}_{2} \mathrm{O}_{3}\right)$, quartzo $\left(\mathrm{SiO}_{2}\right.$-forma hexagonal), cristobalita $\left(\mathrm{SiO}_{2}\right.$-forma tetragonal), cromita aluminosa $\left(\mathrm{Fe}\left(\mathrm{Al}_{3} \mathrm{Cr}\right)_{2} \mathrm{O}_{4}\right)$ e cromatita $\left(\mathrm{CaCrO}_{4}\right)$. Esses resultados são confirmados pelos estudos realizados por Godinho et al. ${ }^{(1)}$

Tabela I. Análise elementar da CIAC considerando alguns elementos selecionados

\begin{tabular}{ccccccccc}
\hline Elemento & $\mathbf{C r}$ & $\mathbf{A l}$ & $\mathbf{F e}$ & $\mathbf{C a}$ & $\mathbf{C}$ & $\mathbf{N}$ & $\mathbf{H}$ & $\mathbf{S}$ \\
\hline [\% em massa] & 59,4 & 3,5 & $\mathrm{I}, 7$ & $\mathrm{I}, 3$ & $0, \mathrm{I}$ & 0,07 & $0, \mathrm{I}$ & 0,3 \\
\hline
\end{tabular}

Tabela 2. Resultados da análise por difração de raios $X$ da CIAC

\begin{tabular}{ccc}
\hline $\begin{array}{c}\text { Afastamento } \\
\text { interplanar, 'd' }[\AA \tilde{A}]\end{array}$ & Mineral & Fórmula \\
\hline 4,05 & cristobalita & $\mathrm{SiO}_{2}$ \\
3,63 & cromatita & $\mathrm{CaCrO}_{4}$ \\
3,35 & quartzo & $\mathrm{SiO}_{2}$ \\
2,66 & eskolaita & $\mathrm{Cr}_{2} \mathrm{O}_{3}$ \\
2,48 & cromita aluminosa & $\mathrm{Fe}\left(\mathrm{Al}_{3} \mathrm{Cr}_{2} \mathrm{O}_{4}\right.$ \\
\hline
\end{tabular}

\subsubsection{Produção da liga em escala de laboratório}

A Tabela 3 resume os resultados mais significativos obtidos nos diferentes experimentos para a produção da liga ferro-cromo a partir da CIAC. A Tabela mostra (com base na massa): a recuperação da fase metálica, a recuperação de cromo na fase metálica e os teores (\% em massa) de cromo, carbono e enxofre na ferroliga obtida. 
Tabela 3. Análise de alguns resultados experimentais significativos

\begin{tabular}{|c|c|c|c|c|c|c|c|}
\hline Tipo & $\begin{array}{c}\text { Ensaio } \\
\mathbf{n}^{\circ}\end{array}$ & $\begin{array}{c}\text { Recuperação da } \\
\text { fase metálica [\%] }\end{array}$ & $\begin{array}{c}\text { Recuperação } \\
\text { de } \mathrm{Cr}[\%]\end{array}$ & $\% \mathbf{C r}$ & $\% \mathrm{C}$ & $\% \mathbf{S}$ & Observações \\
\hline 1 & RED-3 & 52 & 35 & 21,03 & 4,97 & 0,200 & $\begin{array}{l}\text { Fase metálica brilhante. Pouca distinção en- } \\
\text { tre metal e escória. Dificuldade de separação } \\
\text { metal/ escória. Fácil cominuição. }\end{array}$ \\
\hline 2 & RED-5 & 51 & 35 & 21,89 & 9,00 & 0,540 & $\begin{array}{l}\text { Fase metálica brilhante. Pouca distinção en- } \\
\text { tre metal e escória. Dificuldade de separação } \\
\text { metal/ escória. Fácil cominuição. }\end{array}$ \\
\hline 3 & RED-8 & 78 & 69 & 28,11 & 7,00 & 0,350 & $\begin{array}{l}\text { Fase metálica brilhante. Boa distinção entre } \\
\text { metal e escória. Facilidade de separação metal/ } \\
\text { escória. Fácil cominuição. }\end{array}$ \\
\hline \multicolumn{4}{|c|}{ Liga ferro-cromo comercial } & 50,47 & 6,77 & 0,029 & Fase metálica brilhante. Fácil cominuição. \\
\hline
\end{tabular}

Nos primeiros dois tipos de experimentos, a recuperação mais significativa de metal se situou por volta de $50 \%$, enquanto que o teor de cromo na ferroliga se manteve, em média, em $21 \%$. O teor de enxofre, mesmo estando acima do máximo especificado para uma liga comercial, não é tão crítico, uma vez que sua fonte (o agente redutor) pode ser trocada por outra mais adequada. $O$ teor de carbono mostrou-se, em alguns resultados, maior que o da liga comercial, mas, de modo geral, ficou próximo ao esperado pela simulação termodinâmica.

A Tabela 4 destaca alguns dos principais minerais e compostos presentes no experimento RED-5. A existência de minerais, por exemplo, eskolaita, quartzo e cromita aluminosa, deve-se, como mencionado na Tabela 3, de existir eventualmente uma baixa separação entre o metal e a escória - uma vez que estas fases minerais fazem parte da composição da $\mathrm{ClAC}$. A presença dos carbonetos de cromo $(\mathrm{Cr}, \mathrm{Fe})_{7} \mathrm{C}_{3}$ e $(\mathrm{Cr}, \mathrm{Fe})_{23} \mathrm{C}_{6}$ na liga RED-5 pode ser imputada ao seu resfriamento lento, dentro do forno.

Tabela 4. Resultados da análise por difração de raios $X$ da liga RED-5

\begin{tabular}{ccc}
\hline $\begin{array}{c}\text { Afastamento } \\
\text { interplanar, 'd' }[\AA \hat{A}]\end{array}$ & Mineral & Fórmula \\
\hline 3,34 & quartzo & $\mathrm{SiO}_{2}$ \\
2,48 & cromita aluminosa & $\mathrm{Fe}\left(\mathrm{Al}_{3} \mathrm{Cr}\right)_{2} \mathrm{O}_{4}$ \\
2,43 & pirita & $\mathrm{FeS}_{2}$ \\
2,00 & $(\mathrm{Cr}, \mathrm{Fe})_{7} \mathrm{C}$ & $(\mathrm{Cr}, \mathrm{Fe})_{7} \mathrm{C}_{3}$ \\
1,94 & $(\mathrm{Cr}, \mathrm{Fe})_{23} \mathrm{C}_{6}$ & $(\mathrm{Cr}, \mathrm{Fe})_{23} \mathrm{C}_{6}$ \\
$\mathrm{I}, 68$ & eskolaita & $\mathrm{Cr}_{2} \mathrm{O}_{3}$ \\
\hline
\end{tabular}

Um fato digno de consideração, que se tornou claro após os experimentos, é a baixa massa empregada nas cargas. Essa característica, por vezes, dificultou a boa separação entre as fases metal e 'escória', mascarando em algum grau os resultados e dificultando algumas avaliações, por exemplo, a quantificação das frações mássicas das duas fases citadas.

O experimento RED-8 (juntamente com os outros experimentos com cadinhos do tipo 3 ) foi realizado com o intuito de se aumentar a massa da amostra e, simultaneamente, contornar as dificuldades relatadas com o forno de indução. Mas, por limi- tação técnica do novo forno resistivo, teve que ser realizado com a temperatura máxima em torno de $1.300^{\circ} \mathrm{C}$; portanto, $300^{\circ} \mathrm{C}$ mais baixa do que os demais experimentos. Sua carga era constituída de pedaços de briquete contendo cal (preparados para ser a carga do experimento em forno de indução). Da análise dos seus resultados, pode-se afirmar que, em princípio, os fatores briquetagem, uso da cal e maior massa da carga são fatores significativos, que favorecem a redução carbotérmica do cromo contido em CIAC.

De maneira geral, os resultados mostram que não houve uma diferença significativa quanto às variáveis tempo e temperatura empregadas nos experimentos. Contudo, a disposição da carga no cadinho parece ser importante e deve ser avaliada em novos estudos.

\section{CONCLUSÃO}

Tendo por base os experimentos realizados, pode-se afirmar que é possível a elaboração de uma ferroliga $\mathrm{FeCr}-\mathrm{AC}$ a partir de cinzas oriundas do processo de incineração de aparas de couro, por meio da redução carbotérmica nas temperaturas de $1.600^{\circ} \mathrm{C}$ e $1.300^{\circ} \mathrm{C}$.

Este resultado abre novas perspectivas para a reciclagem de resíduos de couro da indústria coureiro-calçadista, pois permite o aproveitamento da energia neles contida e a redução do seu volume sem a criação de um novo resíduo a ser depositado no meio ambiente.

\section{Agradecimentos}

Um dos autores (Claudia Rosane Ribeiro Alves) agradece ao CNPq pelo auxílio, recebido na forma de uma bolsa de mestrado. 


\section{REFERÊNCIAS}

I GODINHO, M.; MARTINS, C. B.; MASOTTI, L.; MARCILIO, N. R.; AZEVEDO, A. Parâmetros operacionais de um gaseificador para resíduos de couro. In: CONGRESSO BRASILEIRO DE ENGENHARIA QUÍMICA (COBEQ), I6., 2006. Anais... São Paulo: ABEQ, 2006.

2 ALVES, C. R. R. Obtenção de uma liga de ferro-cromo de alto teor de carbono a partir das cinzas da incineração de aparas de couro. 2007. 104 p. Dissertação (Mestrado em Tecnologia Mineral/Metalurgia Extrativa) - Engenharia Minas, Metalúrgica e de Materiais, Universidade Federal do Rio Grande do Sul, Porto Alegre, 2007.

3 BENEDUCE NETO, F. Cinética da redução carbotérmica de cromita brasileira (concentrado Jacurici). 2002. 137 p. Tese (Doutorado em Engenharia de Materiais e Metalúrgia) - Escola Politécnica, Universidade de São Paulo, São Paulo, 2002 ,

4 ALVES, C. R. R.; HECK, N. C.; SCHNEIDER, I. A. H. Análise termodinâmica da produção de FeCr-AC a partir das cinzas da incineração de aparas de couro. Tecnologia em Metalurgia e Materiais, v. 5, n. 2, p. 83-8, abr.-jun. 2008.

5 ROSENQVIST, T. Principles of extractive metallurgy. Tokyo: McGraw-Hill, 1974.

6 BALE, C. W.; CHARTRAND, P.; DEGTEROV, S. A.; ERIKSSON, G.; HACK, K.; BEN MAHFOUD, R.; MELANÇON, J.; PELTON, A. D.; PETERSEN, S. FactSage thermochemical software and databases. Calphad, v. 26, n. 2,, p. 189-228, Jun. 2002

7 GÓMEZ-MORENO, F. J.; SANZ-RIVERA, D.; MARTÍN-ESPIGARES, M.; PAPAMELETIOU, D.; SANTI, G. D.; KASPER, G. Characterization of particulate emissions during pyrolysis and incineration of refuse derived fuel. Journal of Aerosol Science, v. 34, n. 9, p. 1267-75, Sept. 2003.

Recebido em: 2/12/2008

Aceito em: 19/09/2009 